\title{
Time to pick the fly's brain
}

\section{Drosophila transformed developmental genetics and cell biology. Now the fruitfly is poised to help biologists decipher how the brain works.}

\section{Claude Desplan}

One afternoon in 1997, a colleague called me into his office to announce that Drosophila research was all washed up. The fruitfly had amassed fantastic successes as a model system for developmental biology. In less than a decade, biologists had used it to map the genetic and molecular network that governs organism development. Now my colleague thought that, having served its purpose, Drosophila must be about to reclaim its status as the odd little creature obscure scientists used to manipu-

late genetic characters. How wrong he was.

In the early 1980s, basic research exploded. Working on Drosophila was valued as a way to understand phenomena believed to be unique to flies, such as weird body deformations. If the Drosophila pioneers believed that their work might have

broader relevance, they could not have dreamed of its awesome implications.

A huge breakthrough came in 1984 with the discovery of the homeobox, the Rosetta Stone of developmental biology. This piece of DNA is shared by the genes that govern body pattern. Mutations in these 'homeotic' genes cause body parts to transform into one another - a leg grows where an antenna should be, four wings develop instead of two, and so on. This homeobox sequence was soon found to have similar functions in mice, humans and indeed most animals.

The discovery transformed vertebrate developmental biology. Biologists identified the molecules underlying what had been abstract concepts, such as morphogenetic gradients - rising or falling amounts of a single protein that direct the development of different body parts. To the surprise of most, it became clear that Drosophila use the same developmental genes as vertebrates. For more than ten years, there was formidable excitement as each new fly gene clarified how a mouse embryo develops, an organ forms or what causes a human mutation.

Things went so fast that by the late 1990 s it seemed that the fruitfly had reached its full potential for answering

big questions, and that developmental biology should be left to those working on vertebrates. By then, fly scientists had made great advances that relied more on clever tricks and a century of genetics than on expensive equipment. A few drosophilists defected to plants or zebrafish in an attempt to adapt these techniques, but the fly stalwarts kept going.

By the start of the new millennium, immensely powerful methods, such as the ability to generate a single mutant cell in an otherwise normal organism, helped solve the major challenge of the time: understanding how cells communicate.

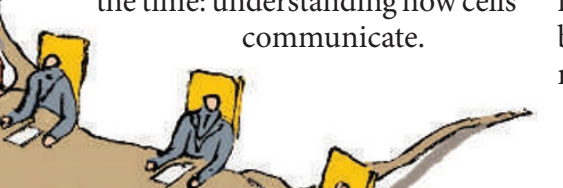

pathways that recognize and then fight bacterial, fungal or viral infections without relying on previous encounters with the pathogen. The same molecules and pathways were later shown to have similar roles in humans, which led to the revival of innate immunity, a field with far-reaching applications in medicine.

What can Drosophila still deliver that will change biology again? The time is ripe to use these tiny creatures to understand the biggest challenge in biology: how the brain works. Flies have a relatively simple brain that controls sophisticated behaviours and can be analysed with machines as bizarre as a fly flight simulator. Drosophila research promises to solve how complex neural circuits in the brain mediate behaviour (see page 193), now that researchers can manipulate single neurons and use sophisticated imaging of the working brain and electrophysiological techniques.

This issue of Nature reports the wholegenome sequences of 12 species of Drosophila, which will allow the organism that can be manipulated so exquisitely to have the best annotated genome. Comparing the genomes of closely and distantly related species will highlight which parts of a protein, or regions of DNA, have

been conserved during evolution, and

Most molecular cascades that bring a signal from the cell surface to the nucleus where it can change the fate of a cell were discovered in flies. Their function was then further analysed in cultured mammalian cells.

In fact, the entire field of cell biology benefited tremendously from work in Drosophila. Logic says processes that concern single cells should be probed in yeast or cultured mammalian cells. But the fly oocyte and embryo proved to be ideal test beds for studying events of the single cell. A purely mechanistic description of molecular interactions was superseded by an understanding of how coordinated events take place in the context of a whole organism.

Immunity is another area in which Drosophila had a surprisingly big role. Findings in flies uncovered the molecular so are likely to have a function. This will offer a way to decipher the grammar of regulatory DNA, which has so far proved elusive.

Powerful genetic tools, genomic advances and beautiful imaging of the tiny structure of a fly brain or embryonic tissue have kept fruitfly research booming for more than two decades. By enabling us to pose biological questions in an in vivo context, the fly is an ideal subject for integrating molecular or cellular processes in the biology of the whole organism. Drosophila research is thriving and should live up to our hopes for many decades to come.

Claude Desplan is in the Department of Biology at New York University, 1009 Silver Center, 100 Washington Square East, New York, New York 10003, USA.

For further reading see www.nature.com/nature. 